\title{
Colorimetric paper-based device for gaseous hydrogen cyanide quantification based on absorbance measurements
}

\author{
João Flávio da Silveira Petruci ${ }^{\mathrm{a}, \mathrm{b}, *}$, Peter C. Hauser ${ }^{\mathrm{a}}$, Arnaldo Alves Cardoso ${ }^{\mathrm{b}}$ \\ a Department of Chemistry, University of Basel, Spitalstrasse 51, 4056 Basel, Switzerland \\ b São Paulo State University (UNESP), Department of Analytical Chemistry, CEP 14800-970, Araraquara, SP, Brazil
}

\section{A R T I C L E I N F O}

\section{Article history:}

Received 31 January 2018

Received in revised form 17 April 2018

Accepted 20 April 2018

Available online 25 April 2018

\section{Keywords:}

Gas sensors

Point-of-care devices

Absorbance

Hydrogen cyanide

Dimethylglyoxime

Miniaturization

\begin{abstract}
A B S T R A C T
Hydrogen cyanide is a highly toxic compound employed for applications such as electroplating, production of organic solvents, fish stunning, and even as a chemical weapon. In this work, we describe a new microfluidic paper-based assay for selective cyanide determination, based on the reaction of the cyanide anion with palladium dimethylglyoximate (DMG) followed by colorimetric reaction of DMG with nickel. Quantification was performed by measuring the absorbance of light passing through the paper, employing an LED emitting at $525 \mathrm{~nm}$ and a log ratio amplifier circuit emulating Lambert-Beer's law. This offers an alternative to the smartphone-based methodologies for signal treatment usually employed in colorimetric $\mu$ PAD-based methods The technique enabled linear calibration within the range $100-500 \mathrm{ppb}$ of $\mathrm{HCN}$, as well as a detection limit of $10 \mathrm{ppb}$ for $\mathrm{HCN}$ in gaseous samples. The sampling time used for this concentration range was $5 \mathrm{~min}$. The method was also tested for the quantification of cyanide in aqueous samples by adding $\mathrm{H}_{3} \mathrm{PO}_{4}$ to the sample, followed by exposure of the paper sensor to the gaseous HCN generated. A detection limit of $1 \mu \mathrm{M}$ was achieved, which makes the method also suitable for detection of cyanide in liquid samples.
\end{abstract}

(ㄷ) 2018 Elsevier B.V. All rights reserved.

\section{Introduction}

Many chemical compounds present in the gas or vapor phases can cause harmful effects to living beings, depending on their occurrence, concentration, reactivity, and toxicity. The development of analytical methods suitable for the detection of molecules in the gas phase in a wide range of matrices still remains a challenge in the analytical chemistry field. New analytical methods must be sufficiently versatile for use in many different applications involving gaseous and other types of samples, in areas such as industrial hygiene, air pollution, detection of biomarkers in exhaled breath, biological greenhouse gas emissions, and determination of dissolved gases or volatile compounds present in liquid solutions (including food, biological, and cosmetics samples) [1-3]. Hydrogen cyanide $(\mathrm{HCN})$ is found in many matrices. It is a highly toxic compound that is naturally present in low quantities in foodstuffs such as apples, mangoes, and rapeseeds, and is used by some plants as a mechanism of defense against herbivores [4]. In industry, cyanide salts are used in electroplating, mining of gold and sil-

\footnotetext{
* Corresponding author at: Department of Chemistry, University of Basel, Spitalstrasse 51, 4056 Basel, Switzerland.

E-mail address: jfpetruci@gmail.com (J.F.d.S. Petruci).
}

ver, metallurgic and photographic activities, and for the production of organic solvents [5]. Hydrogen cyanide is extensively employed as a fumigant in food storage facilities and warehouses [6]. HCN and its derivatives are also used in less desirable activities. In the Philippines, large quantities of $\mathrm{NaCN}$ (estimated at 1 million kilograms since 1960) are deposited into the ocean in order to stun ornamental fish for easier capture [5]. There are also concerns that cyanide may be used as a terrorist weapon. In 1982, cyanide was used as an adulterant in medicine packages in Chicago [7]. In 1995, cyanide was found in subway restrooms in Tokyo [8].

Although the usage of large amounts of cyanide in mining activities is of concern regarding the occupational health of workers, the highest exposures of the general population to HCN are associated with accidental fires, automotive exhaust, and cigarette smoke [9]. Hydrogen cyanide can be lethal to humans following a short period of exposure, which may be by inhalation, absorption through the skin, or ingestion (in the form of cyanide salts). Once absorbed, cyanide limits the functionality of a mitochondrial enzyme responsible for the metabolism of blood oxygen in the electron transport chain process and creation of most of the ATP produced by means of respiration [10]. Cyanide metabolism is very rapid in humans [10], with exposure to high levels of HCN leading to death by suffocation [4]. 
Table 1

Methods for determination of HCN in the gas-phase.

\begin{tabular}{lllll}
\hline Detection Method & $\begin{array}{l}\text { Response } \\
\text { Time }\end{array}$ & LOD & $\begin{array}{l}\text { Reagent } \\
\text { Medium }\end{array}$ & Ref \\
\hline Colorimetric/Naked-eye & $30 \mathrm{~min}$ & $2 \mathrm{ppm}$ & Acetonitrile & {$[28]$} \\
Colorimetric/Transmittance & $2 \mathrm{~min}$ & $0.5 \mu \mathrm{M}$ & Water & {$[8]$} \\
Colorimetric/Conventional & $18 \mathrm{~min}$ & $1 \mathrm{ppm}$ & Acetonitrile & {$[29]$} \\
Colorimetric/Diffuse Reflectance & $10 \mathrm{~s}$ & $1 \mathrm{ppm}$ & Water & {$[33]$} \\
Optical Parameter Oscillator & $10 \mathrm{~s}$ & $0.4 \mathrm{ppm}$ & - & {$[30]$} \\
EPM & $5 \mathrm{~s}$ & $150 \mathrm{ppm}-$ & {$[31]$} \\
QCM & $10 \mathrm{~s}$ & $5 \mathrm{ppm}$ & - & {$[32]$} \\
Colorimetric/Absorbance & $5 \mathrm{~min}$ & $10 \mathrm{ppb}$ & Water & This work \\
\hline
\end{tabular}

EPM: Embedded Piezoresistive Microcantilever; QCM: Quartz Crystal Microbalance.

In healthy adults, blood cyanide (BCN) levels are found in the range from sub- $\mu \mathrm{M}$ up to $40 \mu \mathrm{M}$ [11]. Survivors exposed to smoke from fires showed blood cyanide concentrations of $200 \mu \mathrm{M}$ [8]. Higher levels of BCN are considered toxic and can be fatal. The levels of $\mathrm{CN}^{-}$in drinking water are regulated by many agencies worldwide, such as the World Health Organization (WHO), which limits the presence of cyanide to $2 \mu \mathrm{M}$ [12]. The US EPA and the European Union have established maximum permissible levels of 7.7 and $2 \mu \mathrm{M}$, respectively [5]. In air, a short-term exposure limit of $4.7 \mathrm{ppm}$ of $\mathrm{HCN}$ for $5 \mathrm{~min}$ is recommended by NIOSH. Levels higher than 250 ppm may be lethal within seconds.

Given this background, there is still a need to develop point-ofcare platforms tailored to the determination of hydrogen cyanide in a wide range of samples (gases or liquids). Analytical methods available in the literature for the determination of gaseous HCN cover a broad spectrum of techniques $[4,13,14]$. Despite the availability of many benchtop instrumental methods (notably gas chromatography), there are few examples of simple point-of-care devices suitable for the quantification of sub-ppm or ppb levels of $\mathrm{HCN}$ in the gas phase (Table 1 ). Colorimetric assays are an elegant alternative for detection of an analyte by means of a color change that can indicate the presence or absence of a substance, or provide quantitative data [15]. Many colorimetric reagents have been reported for aqueous cyanide recognition, although their use for direct gaseous $\mathrm{HCN}$ detection is not so common. A disadvantage is that most of the reagents require organic solvents or laborious synthesis steps, substantially reducing their attractiveness.

Colorimetric assays performed on paper-based analytical devices have been used in a wide range of applications involving biological and industrial samples [16,17]. Microfluidic paper-based devices offer many advantages [18], such as (i) low cost and ready worldwide availability; (ii) requirement for small amounts of reagents $(1-10 \mu \mathrm{L})$, avoiding toxic waste generation; (iii) the possibility of POC applications, with high potential for miniaturization; and (iv) excellent performance as solid sorbents for gas collection [19-21]. However, their use for cyanide determination has not yet been fully explored.

Quantification of analytes using paper-based colorimetric assays has often been based on the use of consumer electronic devices (such as smartphones or flatbed scanners) to measure the intensity of light reflected from the surface of the $\mu$ PAD [22]. This detection approach provides a real-time response and a high degree of portability. However, quantification using this approach is not always easy, with a high concentration of the colored product being required, so the limits of detection tend to be high [23]. To address this point, Ellerbee et al. [23] presented an alternative method based on measuring the intensity of the light passing through the paper, rather than the reflected light intensity. The device consisted of a light-emitting diode (LED) as a controlled light source, together with a photodiode detector and integrated amplifier. The associated electronic circuitry was simple and gave an output corresponding to the transmittance $(\mathrm{T})$ parameter.
Here, we propose a new approach for gaseous hydrogen cyanide determination, featuring an improved chemical reaction employing palladium dimethylglyoximate as reagent, followed by a colorimetric reaction step with nickel chloride. To the best of our knowledge, this has only been reported once, without optimization [24]. In addition, measurement of absorbance was used for quantification, employing an LED, photodiode, and log ratio amplifier circuitry. This configuration directly followed Lambert-Beer's law, leading to an output voltage corresponding to the absorbance [25], which, in contrast to the transmittance value, is directly proportional to concentration. The output voltage was measured with a portable digital multimeter, with no need for programming steps or complicated data processing. This versatile and portable platform can be applied for the determination of hydrogen cyanide in gaseous samples and liquid solutions.

\section{Experimental}

\subsection{Materials and reagents}

Microfluidic paper-based analytical devices were produced by the wax printing method, as described elsewhere [26], using a color printer (Model ColorCube 8580, Xerox, New York, United States). Whatman 41 cellulose filter paper (Whatman, Kent, United Kingdom) was used as the solid support. Solutions of potassium cyanide (Synth, Brazil), sodium hydroxide (Synth, Brazil), potassium hydroxide (Qhemis, Brazil), acetic acid (Synth, Brazil), nickel chloride (Synth, Brazil), and potassium chloride (Sigma-Aldrich, St Louis, United States) were prepared using Milli-Q deionized water (Millipore, USA). A solution of dimethylglyoxime(Synth, Brazil) was prepared in methanol (Tedia, Brazil).

\subsection{Instrumentation}

LEDs with emission peaks centered at 525 (HLMP-CM15) and 565 (HLMP-3950) nm were obtained from Broadcom Ltd. (San Jose, California, United States). A sensitive silicon photodiode for the visible spectral range (Model BPW21) was acquired from Osram Opto Semiconductors (Regensburg, Germany). A low noise operational amplifier (OPA 2227U) and a log ratio amplifier (LOG101) were obtained from Texas Instruments (Austin, Texas, United States). Passive components were obtained from Distrelec (Switzerland). Measurements of the output voltage from the LED absorbance detector were made with a digital multimeter (Model M3600, Metex, Toronto, Canada). Absorption spectra were recorded using a conventional UV-vis spectrophotometer (Varian, Santa Clara, California, United States).

\subsection{Preparation of reagent solution and sampling filters}

The palladium (II) dimethylglyoximate $\left(\mathrm{Pd}(\mathrm{DMG})_{2}\right)$ reagent was prepared by precipitation from an acidic solution of palladium chloride by addition of a solution of $1 \%$ dimethylglyoxime in methanol. This reaction mixture was stirred for $24 \mathrm{~h}$. The yellow compound obtained was filtered and then washed sequentially with large amounts of water and methanol. The solution of $\operatorname{Pd}(D M G)_{2}$ was solubilized in $3 \mathrm{~mol} \mathrm{~L}^{-1} \mathrm{KOH}$.

The $\mu$ PADs had a diameter of $8.5 \mathrm{~mm}$, with hydrophobic barriers created using the wax printing method. Subsequently, $2.5 \mu \mathrm{L}$ of PdDMG solution and $2.5 \mu \mathrm{L}$ of $50 \%(\mathrm{v} / \mathrm{v})$ ethylene glycol were added to the paper, prior to exposure to gaseous $\mathrm{HCN}$.

\subsection{Preparation of gaseous standards}

The permeation tube method was employed to generate gaseous standards of hydrogen cyanide used for calibration. The HCN per- 


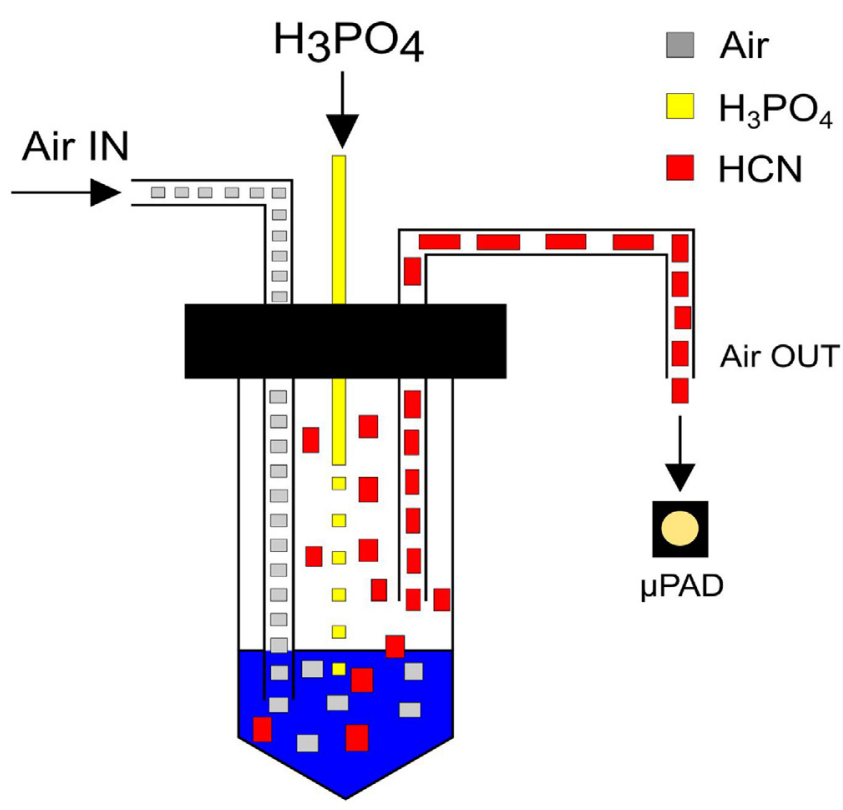

Fig. 1. Scheme of the generation of $\mathrm{HCN}$ from liquid samples containing cyanide anions. Hydrogen cyanide is formed in acid conditions and transported to the $\mu P A D$ using airflow.

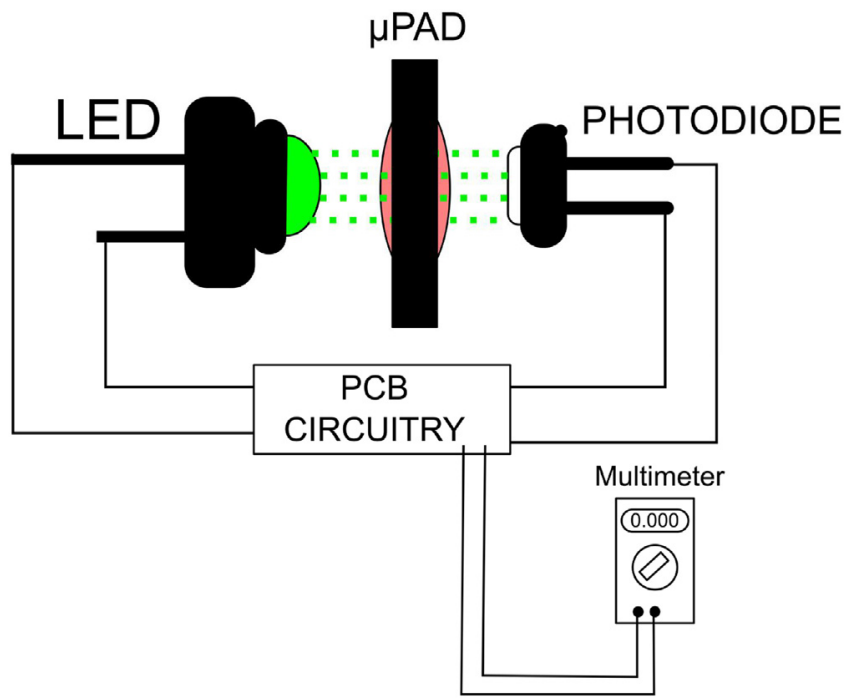

Fig. 2. Diagram of the absorbance based platform for colorimetric assay performed on $\mu$ PAD's.

meation tube (PT) device (VICI Metronics, Santa Clara, United States) was positioned inside a permeation chamber (PC) with the temperature controlled at $30 \pm 0.1^{\circ} \mathrm{C}$. Under this condition, the

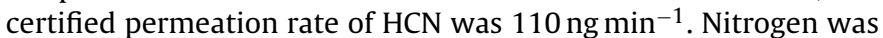
used as the carrier and dilution gases. Mass flow controllers (MFCs) (Model 1179, MKS Instruments, Austin, Texas, United States) were employed to deliver different concentrations of hydrogen cyanide to the sampling stream $(S)$. The gas flow through the permeation chamber was kept at $200 \mathrm{~mL} \mathrm{~min}^{-1}$. The complete standard gas generator is illustrated schematically in Fig. S1 (Supplementary Material).

\subsection{Analytical protocols for gaseous and liquid samples}

For gas samples, the $\mu \mathrm{PAD}$ with $\left[\mathrm{Pd}(\mathrm{DMG})_{2}\right]^{2-}$ and ethylene glycol was positioned under the sampling stream, allowing exposure to $\mathrm{HCN}$. At the end of the sampling time, $2.5 \mu \mathrm{L}$ of a solution con- taining acetic acid and nickel chloride at concentrations of 3 and $1 \mathrm{~mol} \mathrm{~L}^{-1}$, respectively, was added to the paper. The paper was dried for 2 min using a clean air flow and was then positioned in the measurement cell. The absorbance obtained by the LED detector was measured using the digital multimeter.

For aqueous samples, a $2.0 \mathrm{~mL}$ volume of solution containing cyanide was added to a glass tube, which was then capped. A $1 \mathrm{~mL}$ aliquot of $50 \%(\mathrm{v} / \mathrm{v}) \mathrm{H}_{3} \mathrm{PO}_{4}$ was added through a tube located in the top, and the top hole was then closed. Immediately after this step, clean air was manually and gently bubbled through the solution at a constant flow rate, using a $50 \mathrm{~mL}$ syringe that had been previously filled with air. The generated $\mathrm{HCN}$ present in the air was transferred to the $\mu$ PAD surface through a Teflon tube ( $4 \mathrm{~mm}$ i.d.). This procedure was adapted from a published method for determination of cyanide in blood, developed by Ma et al. [8]. Fig. 1 shows the procedure used for the generation and transport of hydrogen cyanide from the liquid sample to the paper sampler device.

\section{Results and discussion}

\subsection{Optimization of detection}

The two chemical reactions selected in this study for the colorimetric detection of cyanide were based on (i) the strong affinity between palladium and cyanide, and (ii) the high molar absorptivity of the nickel dimethylglyoxime complex. Feigl et al. [24] found that in strong basic solutions, the palladium dimethylglyoximate complex reacts selectively with cyanide anions, according to Eqs. (1) and (2).

$$
\begin{aligned}
& \mathrm{Pd}(\mathrm{DMGH})_{2}+2 \mathrm{OH}^{-} \rightarrow \mathrm{Pd}(\mathrm{DMG})_{2}^{2-}+2 \mathrm{H}_{2} \mathrm{O} \\
& \mathrm{Pd}(\mathrm{DMG})_{2}^{2-}+4 \mathrm{CN}^{-} \rightarrow\left[\mathrm{Pd}\left(\mathrm{CN}_{4}\right)\right]^{2-}+2 \mathrm{DMG}^{2-}
\end{aligned}
$$

It is important to note that the soluble palladium dimethylglyoxime complex (formed in Reaction (1)) is highly stable and that other complexation reactions involving the palladium ion (with the exception of cyanide) will not take place. This provides remarkable selectivity of the reagent for cyanide detection. The reaction can be observed visually from decoloration of the yellow solution of $\left[\mathrm{Pd}(\mathrm{DMG})_{2}\right]^{2-}$. As can be seen in Reaction (2), dimethylglyoximate is released from the palladium complex. It is well known [27] that in the $\mathrm{pH}$ range from 5 to 10 , nickel (II) reacts with dimethylglyoxime to form an intense red precipitate (Reaction (3)). The $\mathrm{pH}$ is a fundamental factor, since the Ni-DMG reaction occurs with the protonated form of dimethylglyoxime (DMGH), which has a pKa of 10.14 [27]. Furthermore, highly basic conditions could lead to the formation of $\mathrm{Ni}(\mathrm{OH})_{2}$. Therefore, the analytical signal related to cyanide determination can be based on the formation of this intensely red compound.

$\mathrm{Ni}^{2+}+2 \mathrm{DMGH} \rightarrow \mathrm{Ni}(\mathrm{DMG})_{2}+2 \mathrm{H}^{+}$

The reaction was tested using a paper disk $(8 \mathrm{~mm}$ diameter) treated with $2.5 \mu \mathrm{L}$ of $\left[\mathrm{Pd}(\mathrm{DMG})_{2}\right]^{2-}$ solution $\left(3.4 \mathrm{mmol} \mathrm{L}^{-1}\right)$. To this, $2.5 \mu \mathrm{L}$ of a $\mathrm{CN}^{-}$standard solution at a concentration of $10 \mathrm{mmol} \mathrm{L}^{-1}(25 \mathrm{nmol})$ was added. This was followed by the addition of $2.5 \mu \mathrm{L}$ of a solution containing $3 \mathrm{M}$ acetic acid and $1 \mathrm{M} \mathrm{NiCl}_{2}$. The addition of acetic acid was required in order to lower the $\mathrm{pH}$ and enable formation of the colored complex. The amount used was the experimentally found optimum for maximum color development.

\subsection{Absorbance detection using the microfluidic paper devices}

The detection arrangement used in this study was based on the quantification of absorbance by measuring the light that passed through the $\mu$ PAD impregnated with the colorimetric reagent. The microfluidic analytical paper device $(\mathrm{d}=8.5 \mathrm{~mm})$ was inserted into 


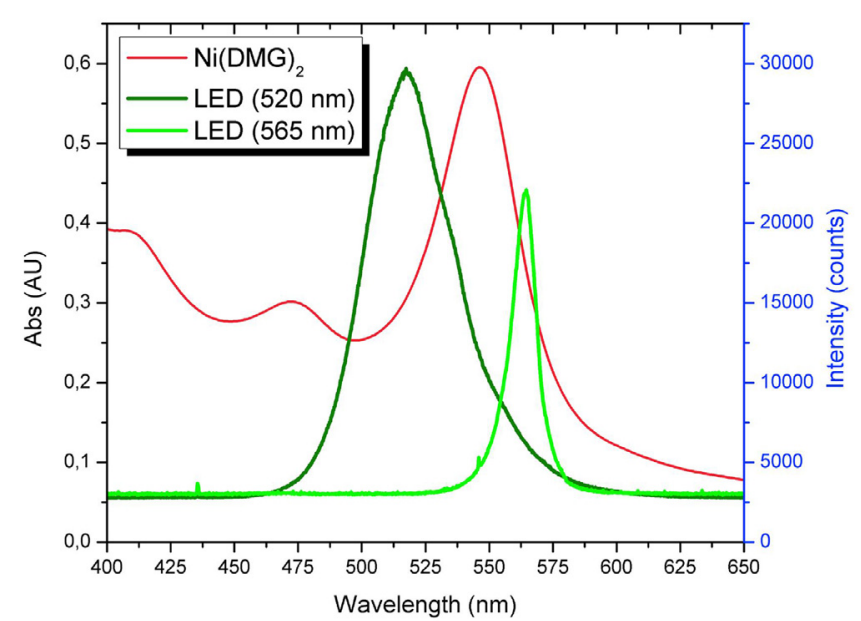

Fig. 3. LED's emission spectra (max. intensity at 525 and $565 \mathrm{~nm}$ ) and absorbance spectrum of $\mathrm{Ni}(\mathrm{DMG})_{2}$. The red precipitate was diluted in water before the spectra acquisition. (For interpretation of the references to colour in this figure legend, the reader is referred to the web version of this article.)

a metal holder $(20(\mathrm{~L}) \times 35(\mathrm{H}) \mathrm{mm})$ and positioned perpendicularly between the LED and the photodiode, hence allowing the transmission of light through the paper, as shown schematically in Fig. 2. The LED and the photodiode were mounted on a support that enabled their alignment on the two sides of the paper. The LED was powered using a constant current of $25 \mathrm{~mA}$, with an LM317 voltage regulator to minimize emission fluctuations. The current generated at the photodiode $(i)$ and the reference current $\left(i_{0}\right)$ provided by an external circuit based on a voltage divider were processed by a log ratio amplifier, producing an output voltage $V_{O}=\log \left(i_{0} / i\right)$, where $1 \mathrm{~V}$ was equivalent to one absorbance unit (AU). An offset circuit was employed to zero the absorbance reading and compensate for imbalance between the intensities of $i$ and $i_{0}$. Additionally, a lowpass filter was applied in order to reduce high frequency noise. The device circuitry is shown in Fig. S2 (Supplementary Information).

As the Lambert-Beer law only holds true for monochromatic radiation, the use of LEDs as light sources in absorbance measurements could cause deviation from linearity. For nonmonochromatic sources, this deviation can be minimized by selecting an LED whose emission spectrum satisfactorily matches the absorbance bands [25]. In this study, two LED options were investigated (Fig. 3). In order to evaluate linearity, the absorbance of the $\mathrm{Ni}(\mathrm{DMG})_{2}$ generated with different concentrations of cyanide was measured using the two LEDs with emission peaks centered at 525 and $565 \mathrm{~nm}$. It was found that the analytical signal was linear when the LED emitting at $525 \mathrm{~nm}$ was employed (Fig. 4).

\subsection{Effect of drying of the paper device on the signal}

Due to the small volumes of sample and reagent solutions employed, evaporation of water from the paper devices could not be neglected. As shown in Fig. 4, the analytical signal was strongly influenced by the moisture content of the paper. This could be explained by light scattering related to changes in the refractive index of the paper-fluid matrix, resulting in a pronounced effect on the light transmitted through the paper. At the start of the experiments, the absorbance was zeroed using the offset circuitry, employing a freshly prepared blank paper containing only $\left[\mathrm{Pd}(\mathrm{DMG})_{2}\right]^{2-}$ and $\mathrm{NiCl}_{2}$. When the paper was still visibly wet, the stability was relatively poor, with precipitate formation also contributing to the low stability of the analytical signal in the initial minutes. The signal varies and increases continuously while the paper is drying. A stable reading was only reached when the paper

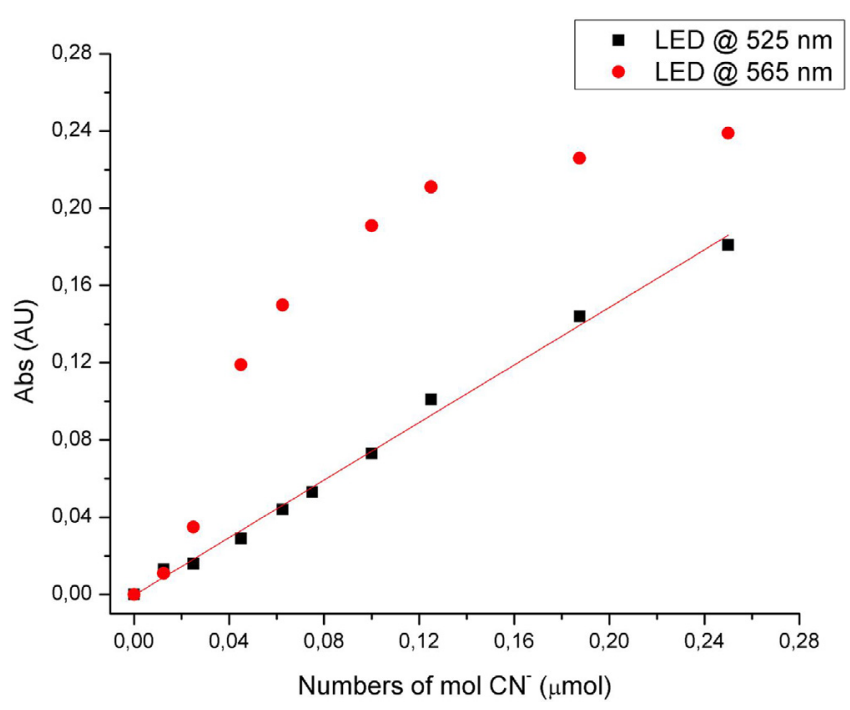

Fig. 4. Relation of the analytical signal versus number of mols of cyanide using LED's emitting at 525 and $565 \mathrm{~nm}$.

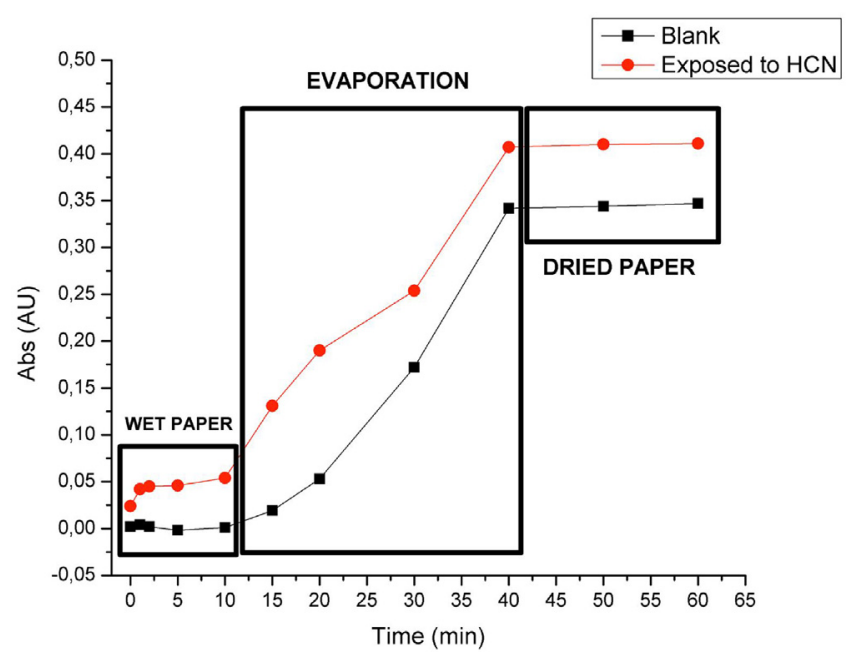

Fig. 5. Effect of paper moisture on the absorbance measurements of the colorimetric assay of the blank ([Pd(DMG $\left.)_{2}\right]^{2-}$ ) and exposed to HCN.

was dry, indicating that the measurement should be carried out under this condition (Fig. 5). A disadvantage was the long time required for drying the paper. This was resolved by using a flow of air (provided by a hair dryer) to accelerate the drying process, which reduced the time needed to reach the steady state from 40 to $2 \mathrm{~min}$. The stability of the absorbance after $2 \mathrm{~min}$ of exposure to the air flow is shown in Fig. 6.

\subsection{Calibration and limit of detection for the direct determination of cyanide in solution}

In order to evaluate the sensitivity of the method, a calibration curve was constructed of the absorbance according to the amount of $\mathrm{CN}^{-}$(in nmol). For each measurement, an average of three independent replicates was used. Linearity was obtained in the range from 6.75 to $25 \mathrm{nmol}$ of $\mathrm{CN}^{-}$, described by the equation: $\mathrm{A}=0.739$ $\mathrm{CN}^{-}+7.48 \times 10^{-4}\left(\mathrm{r}^{2}>0.99\right)$. The precision of the measurements, determined as the relative standard deviation (RSD), was $4.25 \%$. The limit of detection was $2 \mathrm{nmol}$ (based on three times the standard deviation of the blank signal). 
Table 2

Analytical parameters of $\mathrm{HCN}$ determination.

\begin{tabular}{|c|c|c|}
\hline Parameter & HCN gaseous samples & HCN liquid samples \\
\hline Linear range & $100-500 \mathrm{ppb}$ & $5-100 \mu \mathrm{M}$ \\
\hline Correlation coefficient & 0.991 & 0.993 \\
\hline Limit of detection ( $3^{*}$ SD of blank) & $10 \mathrm{ppb}$ & $1 \mu \mathrm{M}$ \\
\hline Calibration equation & $\mathrm{A}=1.22 \times 10^{-4}[\mathrm{HCN}]-0.0275$ & $\mathrm{~A}=0.739\left[\mathrm{CN}^{-}\right]+7.48 \times 10^{-4}$ \\
\hline Response time & $5 \mathrm{~min}$ & - \\
\hline Precision (RSD, $\mathrm{n}=7$ ) & $4.25 \%$ & $7.5 \%$ \\
\hline
\end{tabular}

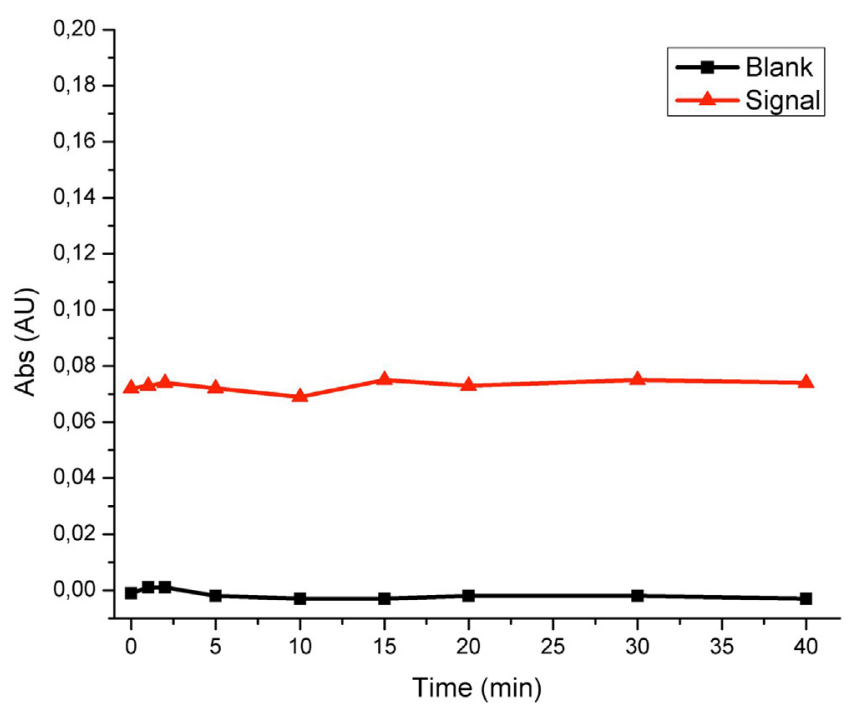

Fig. 6. Signal stability after drying of the $\mu P A D$ with airflow for $2 \mathrm{~min}$.

\subsection{Optimization and calibration of the sensor for determination of gaseous HCN}

Evaluation was made of the conditions necessary to improve the efficiency of HCN sampling and maximize the performance of the sensing system. Increasing the efficiency of the sensing layer for trapping molecules was essential for optimizing the conditions of the method. The sample flow was passed over the $\mu$ PAD containing $\mathrm{Pd}(\mathrm{DMG})_{2}$ and ethylene glycol (ETG) for $5 \mathrm{~min}$, followed by the colorimetric reaction with acetic acid and nickel chloride, and exposure to the air flow for $2 \mathrm{~min}$. The HCN concentration was kept at $500 \mathrm{ppb}$ and the gas flow rate was adjusted in the range from 50 to $200 \mathrm{~mL} \mathrm{~min}^{-1}$. The absorbance of the sample was then measured after zeroing the absorbance using the offset circuitry and the paper containing the Pd complex/acetic acid/nickel chloride mixture. The best sampling efficiency was obtained at a flow rate of $200 \mathrm{~mL} \mathrm{~min}^{-1}$, which was therefore used in all subsequent experiments.

Previous studies [19-21] found that the addition of ETG to reagents resulted in improved gas sampling efficiency. In this study, it was demonstrated that the humidity of the paper affected the analytical signal response. Therefore, the presence of ETG could lead to an improvement of the sampling efficiency, but could also affect the stability of the analytical signal. A four-fold increase of the absorbance was achieved when the $\mu P A D$ was impregnated with $2.5 \mu \mathrm{L}$ of $50 \%(\mathrm{v} / \mathrm{v})$ ethylene glycol, which was therefore chosen as the optimum condition.

The sensor was calibrated by determining the absorbance as a function of the $\mathrm{HCN}$ concentration. The sampling flow rate was maintained at $200 \mathrm{~mL} \mathrm{~min}^{-1}$, with a sampling time of $5 \mathrm{~min}$. After the sampling step, $2.5 \mu \mathrm{L}$ of solution containing acetic acid and nickel chloride was added to the $\mu \mathrm{PAD}$, followed by exposure to the air flow for $2 \mathrm{~min}$. A linear relation was obtained in the range from 100 to $500 \mathrm{ppb}$ of HCN. For each concentration, an average of three replicates was calculated. The linear equation obtained was as follows:

$\mathrm{A}=1.22 \times 10^{-4}[\mathrm{HCN}]-0.0275$

Under the experimental conditions employed, the RSD of the measurements $(n=7)$ was $4.25 \%$. Based on three times the standard deviation of the blank signal, the calculated limit of detection was $10 \mathrm{ppb}$. The sensor performance parameters are summarized in Table 2. It is important to mention that the limit of detection and linear range were obtained for a sampling time of $5 \mathrm{~min}$ and could be modified according to the application requirements.

\subsection{Determination of cyanide in liquid solution by volatilization}

Volatilization of compounds present in liquid solutions is a possible cleanup procedure for minimizing interference from the liquid matrix. In this study, HCN was released from liquid solution using an adaption of the procedure described by Ma et al. [8]. The analytical procedure consisted of the formation of gaseous hydrogen cyanide after addition of $1.0 \mathrm{~mL}$ of $50 \%(\mathrm{v} / \mathrm{v})$ phosphoric acid to the sample. Air was then bubbled through the solution using a syringe, producing an output air stream enriched in $\mathrm{HCN}$. The $\mu$ PAD containing $\left[\mathrm{Pd}(\mathrm{DMG})_{2}\right]^{2-}$ and ETG was positioned at the end of the output air stream for exposure to the gaseous HCN. Using this procedure, a linear relation was obtained for aqueous cyanide in the range from 5 to $100 \mu \mathrm{mol} \mathrm{L}^{-1}$, with a limit of detection of $1 \mu \mathrm{mol} \mathrm{L}{ }^{-1}$. It is important to mention that the limit of detection can be adaptable (e.g. by increasing sample volume or sampling time), depending on the requirements of the application.

\section{Conclusions}

A portable, low cost, simple, and highly selective and sensitive optical platform for the determination of gaseous HCN was developed, based on absorbance measurements using a microfluidic paper analytical device. The $\mathrm{Pd}(\mathrm{DMG})_{2}$ reagent could be easily synthesized in simple steps using readily available chemicals. The use of a log ratio amplifier circuit (LOG101) enabled direct measurements of absorbance through the paper, representing a robust yet inexpensive alternative to commonly used approaches such as the employment of smartphone cameras or similar devices for colorimetric assays performed on $\mu$ PADs. An inexpensive LED emitting at $525 \mathrm{~nm}$ and a silicon photodiode were employed in the detection device. The use of paper as the solid support offered the advantages of disposability, low cost, easy handling, use of small volumes of reagents, and minimal environmental impacts. The platform was able to detect gaseous HCN and cyanide in liquid solution with limits of detection of $10 \mathrm{ppb}$ and $1 \mu \mathrm{mol} \mathrm{L}^{-1}$, respectively, under the conditions employed in this study.

The sensing device showed excellent performance when compared to other optical arrangements for detection of gaseous HCN described in the literature. The method is suitable for the analysis of hydrogen cyanide in air (such as indoor environments), in biological samples (such as blood), and in aqueous media. Further development of the sampling unit, involving use of portable low- 
power air pumps and valves controlled by computer, should enable production of a fully automated device for in situ determination of $\mathrm{HCN}$ in various situations.

\section{Acknowledgments}

JFSP and AAC are grateful for financial support provided by the São Paulo State Research Foundation (FAPESP, grants 2016/16830-0 and 2015/23265-5).

\section{Appendix A. Supplementary data}

Supplementary data associated with this article can be found, in the online version, at https://doi.org/10.1016/j.snb.2018.04.101.

\section{References}

[1] J. Hodgkinson, R.P. Tatam, Optical gas sensing: a review, Meas. Sci. Technol. 24 (2013) 12004

[2] C. Wang, P. Sahay, Breath analysis using laser spectroscopic techniques: breath biomarkers, spectral fingerprints, and detection limits, Sensors 9 (2009) 8230-8262.

[3] Á. Bálint, S. Hoffmann, A. Anton, T. Szili-Kovács, G. Heltai, Contribution of agricultural field production to emission of greenhouse gases (GHG), Ecol. Chem. Eng. S 20 (2013) 233-245.

[4] E.P. Randviir, C.E. Banks, The latest developments in quantifying cyanide and hydrogen cyanide, TrAC - Trends Anal. Chem. 64 (2015) 75-85.

[5] J. Ma, P.K. Dasgupta, Recent developments in cyanide detection: a review, Anal. Chim. Acta 673 (2010) 117-125.

[6] H.G. Higson, A review of the methods available for the detection and determination of small amounts of cyanide, Analyst 88 (1963) 751-760.

[7] M. Eckstein, Enhancing public health preparedness for a terrorist attack involving cyanide, J. Event Manag. Syst. 35 (2008) 59-65.

[8] J. Ma, S.I. Ohira, S.K. Mishra, M. Puanngam, P.K. Dasgupta, S.B. Mahon, M. Brenner, W. Blackledge, G.R. Boss, Rapid point of care analyzer for the measurement of cyanide in blood, Anal. Chem. 83 (2011) 4319-4324.

[9] T.I. Mudder, M.M. Botz, E.C. Engineering, G. Lane, Cyanide and society: a critical review, Eur. J. Min. Proc. Environ. Protect. 4 (2004) 62-74.

[10] A.E. Lindsay, A.R. Greenbaum, D.O. Hare, Analytical techniques for cyanide in blood and published blood cyanide concentrations from healthy subjects and fire victims, Anal. Chim. Acta 511 (2004) 185-195.

[11] K. Tsuge, M. Kataoka, Y. Seto, Y. Cyanide, Thiocyanate levels in blood and saliva of healthy adult volunteers, J. Health Sci. 46 (2000) 343-350.

[12] Y. Sun, Y. Liu, W. Guo, Fluorescent and chromogenic probes bearing salicylaldehyde hydrazone functionality for cyanide detection in aqueous solution, Sens. Actuators B Chem. 143 (2009) 171-176.

[13] P.A. Smith, M.V. Sheely, T.A. Kluchinsky Jr., Solid phase microextraction with analysis by gas chromatography to determine short term hydrogen cyanide concentrations in a field setting, J. Sep. Sci. (2002) 917-921.

[14] J.I. Baumbach, Ion mobility spectrometry coupled with multi-capillary columns for metabolic profiling of human breath, J. Breath Res. 3 (2009) 34001.

[15] L.F. Capitán-Vallvey, A.J. Palma, Recent developments in handheld and portable optosensing - a review, Anal. Chim. Acta 696 (2011) 27-46.

[16] D.D. Liana, B. Raguse, J.J. Gooding, E. Chow, Recent advances in paper-based sensors, Sensors (2012) 11505-11526

[17] Y. Yang, E. Noviana, M.P. Nguyen, B.J. Geiss, D.S. Dandy, C.S. Henry, Paper-based microfluidic devices: emerging themes and applications paper-based microfluidic devices: emerging themes and applications, Anal. Chem. 89 (2017) 71-91.

[18] G.M. Whitesides, The origins and the future of microfluidics, Nature 442 (2006) 368-373.

[19] J.F.S. Petruci, A.A. Cardoso, Sensitive luminescent paper-based sensor for the determination of gaseous hydrogen sulfide, Anal. Methods 7 (2015) 2687-2692.
[20] J.F.S. Petruci, A.A. Cardoso, Portable and disposable paper-based fluorescent sensor for in situ gaseous hydrogen sulfide determination in near real-time, Anal. Chem. 88 (2016) 11714-11719.

[21] G. Garcia, A.G. Allen, A.A. Cardoso, A. New, A new and simple visual technique based on indigo dye for determination of ozone in ambient air, Water Air Soil Pollut. 225 (2014) 1836.

[22] A. Roda, E. Michelini, M. Zangheri, M. Di, D. Calabria, P. Simoni, Smartphone-based biosensors: a critical review and perspectives, Trends Anal. Chem. 79 (2016) 317-325.

[23] A.K. Ellerbee, S.T. Phillips, A.C. Siegel, K.A. Mirica, A.W. Martinez, P. Striehl, N. Jain, M. Prentiss, G.M. Whitesides, Quantifying colorimetric assays in paper-Based microfluidic devices by measuring the transmission of light through paper, Anal. Chem. 81 (2009) 8447-8452.

[24] F. Feigl, H.E. Feigl, On the reactivity of innercomplex-bonded palladium, Anal. Chim. Acta 3 (1949) 300-309.

[25] D.A. Bui, P.C. Hauser, Analytical devices based on light-emitting diodes - a review of the state-of-the-art, Anal. Chim. Acta 853 (2015) 46-58.

[26] D.M. Cate, J.A. Adkins, J. Mettakoonpitak, C.S. Henry, Recent developments in paper-based microfluidic devices, Anal. Chem. 87 (2015) 19-41.

[27] M.A. Bambenek, R.T. Pflaum, The reaction of nickel with dioximes, Inorg. Chem. 758 (1963) 24-27.

[28] R. Gotor, A.M. Costero, S. Gil, M. Parra, R. Martinez-Mañez, F. Sancenon, P. Gaviña, Selective and sensitive chromogenic detection of cyanide and HCN in solution and in gas phase, Chem. Commun. 3 (2013) 3-5.

[29] T. Abalos, S. Royo, R. Martinez-Mañez, F. Sancenon, J. Soto, A.M. Costeiro, S. Gil, M. Parra, Surfactant-assisted chromogenic sensing of cyanide in water, New J. Chem. (2009) 1641-1645.

[30] D.D. Arslanov, M.P.P. Castro, A.H. Neerincx, S.M. Cristescu, F.J.M. Harren, Optical parametric oscillator-based photoacoustic detection of hydrogen cyanide for biomedical applications, J. Biomed. Opt. 18 (2013) 107002.

[31] T.L. Porter, T.L. Vail, M.P. Eastman, R. Stewart, J. Reed, R. Venedam, W. Delinger, A solid-state sensor platform for the detection of hydrogen cyanide gas, Sens. Actuators B Chem. 123 (2007) 313-317.

[32] M. Yang, J. He, X. Hu, C. Yan, Z. Cheng, CuO nanostructures as quartz crystal microbalance sensing layers for detection of trace hydrogen cyanide gas, Environ. Sci. Technol. 45 (2011) 6088-6094.

[33] L.A. Greenawald, J.L. Snyder, N.L. Fry, M.J. Sailor, G.R. Boss, H.O. Flinkea, S. Bell, Development of a cobinamide-based end-of-service-life indicator for detection of hydrogen cyanide gas, Sens. Actuators B 221 (2015) 379-385.

\section{Biographies}

João Flávio da Silveira Petruci obtained his Ph.D in Analytical Chemistry at São Paulo State University (UNESP), Araraquara, Brazil, in 2014. Currently, he has been working as a postdoctoral fellow at UNESP. He was a visiting researcher at the University of Ulm, Germany (2013 and 2015) and the University of Basel, Switzerland (2017). His research interests involve gas sensors, analytical chemistry, spectroscopy, miniaturized and portable platforms, instrumentation, and optical sensor technology.

Peter C. Hauser is Associate Professsor in Analytical Chemistry since 1996, at University of Basel, Switzerland. He is member of the Editorial Advisory Boards of 'Analytica Chimica Acta' (since 2002) and 'Electrophoresis' (since 2004). Over the years, he has acquired a broad range of expertise in diverse fields such as fuel cells, the spectroscopic methods of the ICP and XPS, flow-injection analysis, sensors and the separation methods of HPLC and capillary electrophoresis, and lab-on-chip devices. Current Research Interests include the following topics: miniaturization in analytical chemistry, micro- separation techniques, electrochemical detection techniques for lab-on-chip and conventional capillary electrophoresis, optoelectronic detectors, automation in analytical chemistry, electrochemical gas sensors, miniaturized plasma spectrometers and detectors, clinical analysis, field and process analysis.

Arnaldo Alves Cardoso obtained his Ph.D in Analytical Chemistry in 1991, at University of São Paulo, Brazil. He worked as visiting researcher at Texas Tech University in 1995. Currently, he is full professor in analytical chemistry at São Paulo State University (UNESP). His research interests involve Environmental Chemistry, Particulate Material, Analytical Methods, Gas sensors and spectroscopy. 